Local Structural Models of Complex Oxygen- and Hydroxyl-rich $\mathrm{GaP} / \mathrm{InP}(001)$ Surfaces

B. Wood, T. Ogitsu, E. Schwegler

November 7, 2011

Journal of Chemical Physics 
This document was prepared as an account of work sponsored by an agency of the United States government. Neither the United States government nor Lawrence Livermore National Security, LLC, nor any of their employees makes any warranty, expressed or implied, or assumes any legal liability or responsibility for the accuracy, completeness, or usefulness of any information, apparatus, product, or process disclosed, or represents that its use would not infringe privately owned rights. Reference herein to any specific commercial product, process, or service by trade name, trademark, manufacturer, or otherwise does not necessarily constitute or imply its endorsement, recommendation, or favoring by the United States government or Lawrence Livermore National Security, LLC. The views and opinions of authors expressed herein do not necessarily state or reflect those of the United States government or Lawrence Livermore National Security, LLC, and shall not be used for advertising or product endorsement purposes. 


\title{
Local structural models of complex oxygen- and hydroxyl-rich GaP/InP(001) surfaces
}

\author{
Brandon C. Wood, Tadashi Ogitsu, and Eric Schwegler \\ Quantum Simulations Group, Lawrence Livermore National Laboratory, Livermore, CA 94550
}

\begin{abstract}
We perform density-functional theory calculations on model surfaces to investigate the interplay between the morphology, electronic structure, and chemistry of oxygen- and hydroxyl-rich surfaces of $\operatorname{InP}(001)$ and $\mathrm{GaP}(001)$. Four dominant local oxygen topologies are identified based on the coordination environment: $M-\mathrm{O}-M$ and $M-\mathrm{O}-\mathrm{P}$ bridges for the oxygen-decorated surface; and $M-[\mathrm{OH}]-M$ bridges and atop $M-\mathrm{OH}$ structures for the hydroxyl-decorated surface $(M=\mathrm{In}, \mathrm{Ga})$. Unique signatures in the electronic structure are linked to each of the bond topologies, defining a map to structural models that can be used to aid the interpretation of experimental probes of native oxide morphology. The $M-\mathrm{O}-M$ bridge can create a trap for hole carriers upon imposition of strain or chemical modification of the bonding environment of the $M$ atoms, which may contribute to the observed photocorrosion of $\mathrm{GaP} / \mathrm{InP}$-based electrodes in photoelectrochemical cells. Our results suggest that a simplified model incorporating the dominant local bond topologies within an oxygen adlayer should reproduce the essential chemistry of complex oxygen-rich $\operatorname{InP}(001)$ or $\mathrm{GaP}(001)$ surfaces, representing a significant advantage from a modeling standpoint.
\end{abstract}

\section{INTRODUCTION}

Photoelectrochemical production of hydrogen using sunlight and water represents an attractive approach for environmentally benign chemical storage of solar en$\mathrm{ergy}^{1}$. However, optimizing semiconductor-based photoelectrode materials for hydrogen evolution has proven extremely difficult, in part because the microscopic details of the chemical processes active on the exposed photoelectrode remain poorly understood, as does the connection of these processes to the specific electronic and atomic structures of the semiconductor surface and interface $^{2-4}$. This is further complicated by the range of materials properties that must be simultaneously satisfied, including a band gap matched to the visible spectrum; proper band-edge alignment for driving the water redox reaction at the interface; good carrier mobility; stability under operating conditions in an electrolyte solution; and potential for catalyzing the surface water-splitting reaction ${ }^{1,3}$.

At present, the most efficient photoelectrochemical devices are based on III-V semiconductor photocathodes, with the highest rates of hydrogen generation achieved using a polar (001) surface of $\mathrm{Ga}_{x} \mathrm{In}_{1-x} \mathrm{P}^{5}$. Unfortunately, efficiency comes at the expense of short device lifetime due to fast degradation of the electrode ${ }^{6-12}$. In order to tune stability and reactivity, a better understanding of the morphology and composition of the semiconductor surface under realistic environmental conditions is required; structural features can then be linked to specific electronic properties relevant to photocatalysis and photocorrosion. Of particular interest is the role of surface oxygen and hydroxyl, which are known to be present in high concentrations on exposed polar surfaces of many III-V semiconductors but are often neglected in idealized models, in part due to the complexity of the surface morphology. It has been suggested that the stability of some III-V surfaces in an electrolyte, as well as the photocatalytic water-splitting activity itself, may be tied to the existence of this surface oxygen ${ }^{9,11,13-16}$. Such a connec- tion between water splitting and surface oxygen is also consistent with our initial results on the $\operatorname{InP}(001)$-water interface $^{17}$.

In this work, we aim to provide a detailed description of the interaction of chemisorbed oxygen and hydroxyl with model III-V semiconductor photocathodes, and of the nucleation of surface oxide and hydroxide films from component elements. Since alloys of $\mathrm{GaP}$ and $\mathrm{InP}$ demonstrate the highest overall conversion efficiency of any known material, we have focused our study on (001) surfaces of these two materials, which individually have known water-splitting activity ${ }^{5,16,18-22}$ and represent endpoints in the $\mathrm{Ga}_{x} \mathrm{In}_{1-x} \mathrm{P}$ composition phase diagram. In addition, GaP and $\mathrm{InP}$ are widely studied semiconductor materials with key applications in optoelectronics and photovoltaics. Accordingly, understanding the nature of adsorbed oxygen on these surfaces is of further importance to these communities. To this end, we have performed extensive total-energy calculations based on density functional theory to explore the structure, stability, and chemistry of oxygen- and hydroxyl-rich $\operatorname{InP}(001)$ and $\mathrm{GaP}(001)$ surfaces.

Although dissociative chemisorption of molecular oxygen is known to take place at III-V surfaces at ambient temperature $^{23}$, experiments suggest that different local thermal and electrochemical growth conditions lead to significant variation in the composition and morphology of the resulting native oxide films ${ }^{9,10,24-30}$. The discrepancies point to a complex free energy surface featuring competing variants whose expression is determined by a combination of kinetics and thermodynamics. These may mix regionally within a single sample, or else form the structural motifs of an amorphous oxide layer. Since these complexities make specific determination of a single dominant oxide structure difficult, the goal of this work is to identify those structural motifs that are most likely to exist in real oxide layers on $\operatorname{InP}(001)$ and $\mathrm{GaP}(001)$. As we will demonstrate, similar dominant local bond topologies are evident across all of our tested surfaces, and can be related to local geometries within bulk oxides derived 
from In, Ga, and P. Our calculation method permits us to isolate the corresponding electronic signatures and relative stability of each topology. Notably, many of these signatures should be reflected in surface-sensitive spectroscopic measurements.

\section{METHODS}

Results are obtained using density functional theory and a plane-wave basis set, as implemented in the Quantum-Espresso code $^{31}$. The Perdew-BurkeErnzerhof (PBE) exchange-correlation functional was used $^{32}$. Ultrasoft pseudopotentials ${ }^{33}$ were employed with plane-wave and charge-density cutoffs of 30 and $240 \mathrm{Ry}$, respectively, and with semi-core $d$ states included in the valence descriptions for In and Ga. We inserted $12 \AA$ of vacuum separation between periodic slab images. Ninelayer slabs exposing the (001) surface were used, with the odd number to ensure both surfaces were In/Garich. The bottom surface was terminated with a hydrogen bridging every other pair of metal atoms along [110], plus an additional atop hydrogen on every metal atom. This choice of surface termination was motivated by the results of Ref. ${ }^{34}$, where exact charge compensation was found to be necessary for adequate passivation of the surface of a layered polar structure. Tests confirmed that the corresponding surface states lay inside the band edges. All atoms were relaxed independently except for the bottom two semiconductor layers, which were fixed to the bulk geometry, and the hydrogen termination layer. A $6 \times 6 \mathrm{k}$-point mesh was used in the slab plane for the $(2 \times 2)$ surfaces, with one $\mathbf{k}$-point perpendicular to the slab. For the other surface reconstructions, the in-plane k-point mesh was adjusted to maintain the same density.

Three sets of surface models are used to explore different coverages and morphologies for $\mathrm{O} / \mathrm{OH}$ adsorption: a $M$-rich mixed-dimer $\delta(2 \times 4)$ reconstruction $(M=\mathrm{In}, \mathrm{Ga})$; a regular rectangular lattice of $M-M$ dimers in a $(1 \times 2)$ reconstruction; and a collection of $(2 \times 2)$ surfaces generated by relaxing initially unreconstructed samples under high $\mathrm{O} / \mathrm{OH}$ loading. Defining the surface coverage $\theta$ as the ratio of adsorbates to pre-relaxation surface atoms, these three correspond to $\theta=0.1, \theta=0.5$, and $\theta=1$, respectively.

The choice of surface reconstruction for the dilute coverage limit $(\theta=0.1)$ was motivated by the observation that the two most common reconstructions of $\operatorname{InP}(001)$ and $\mathrm{GaP}(001)$ under ambient conditions are the $M$-rich mixed-dimer $\delta(2 \times 4)$ and the P-rich $(2 \times 1)$, depending on the specific growth environment ${ }^{35-43}$. Of these, experiments on $\operatorname{InP}(001)$ have shown that the P-rich surface features stable $\mathrm{P}-\mathrm{P}$ dimers that exhibit weak oxygen uptake at ambient temperature, whereas the $M$-rich mixed-dimer $\delta(2 \times 4)$ surface reacts readily with oxygen ${ }^{23}$. Since we are interested in surface oxygen binding, we therefore consider only the $M$-rich surface in the present study. The $M$-rich mixed-dimer $\delta(2 \times 4)$ surface has also been observed in the ternary $\operatorname{InGaP}(001)$ alloy ${ }^{44}$. It is worth mentioning that theoretical $a b$ initio studies have predicted an additional surface Ga-dimer $(2 \times 4)$ reconstruction of $\mathrm{GaP}(001)$ for very Ga-rich growth environments ${ }^{35,37,38,42}$; however, as this has not been realized experimentally, we restrict our study to the mixed-dimer $\delta(2 \times 4)$ reconstruction found in both materials. The topologically distinct high-symmetry sites we tested are shown in Fig. 1.

Dense oxygen and hydroxyl coverage $(\theta=1)$ was also considered in order to better model the initial stages of native oxide/hydroxide nucleation upon higher oxygen or hydroxyl loading. Recognizing that $\mathrm{InP} / \mathrm{GaP}(001)$ surfaces could exhibit a very different reconstruction under such conditions, model structures were generated by placing oxygen atoms and hydroxyl groups in highsymmetry sites of initially unreconstructed $M$-rich $(2 \times 2)$ surface slabs, from which atoms were allowed to relax to local-minimum configurations. Atop, hollow, and both bridge sites were tested (see panel inset, Fig. 2). In each case, the four adsorbate positions were perturbed independently to generate as many unique surfaces as possible. Although not an exhaustive search of the parameter space, this method enabled generation of seven additional oxygen-rich and three additional hydroxyl-rich surface structures, shown in Fig. 2. Since the final configurations do not always reflect the initial site symmetry, we avoid confusion by adopting a notation that indexes surface morphologies with a letter corresponding to the initial adsorption site ( $A=$ atop, $X=$ bridge $\mathrm{x}, Y=$ bridge $\mathrm{y}$, $H=$ hollow). An additional numerical index is appended to distinguish the various configurations for the $A$ and $H$ symmetry sites in the case of the oxygen-rich surfaces in Fig. 2a.

Last, a regular rectangular lattice of $M-M$ dimers in a $(1 \times 2)$ pattern $(\theta=0.5)$ was included, as this is the simplest representative model for $\mathrm{InP} / \mathrm{GaP}(001)$ surface dimerization. Such dimerization is the dominant characteristic not only of the mixed-dimer $\delta(2 \times 4)$ reconstruction, but also in additional competing (001) surface reconstructions in $M$-rich environments, such as those expressed under non-equilibrium growth conditions where kinetics are expected to dominate ${ }^{45}$. Here, oxygen was tested in the surface bridge y site and in a subsurface site between $M$ and P; hydroxyl adsorption was tested in the surface bridge y site and at the $M$ atop site (see panel inset, Fig. 2 for designations of high-symmetry sites). We find that in many instances, this simplified model based on a lattice of $M-M$ dimers captures much of the physics of the more complex surfaces due to the local nature of the oxygen electronic signature.

Adsorption energies were calculated as $\Delta E=$ $E_{\text {surf }+ \text { ads }}-E_{\text {surf }}-E_{\text {ads }}$, where $E_{\text {surf+ads }}, E_{\text {surf }}$, and $E_{\text {ads }}$ are the reference energies of the adsorbed system, clean surface, and gas-phase adsorbate $(\mathrm{O}$ or $\mathrm{OH})$, respectively. For dilute adsorption on the mixed-dimer $(2 \times 4)$ reconstruction, the reference for the clean surface is the re- 


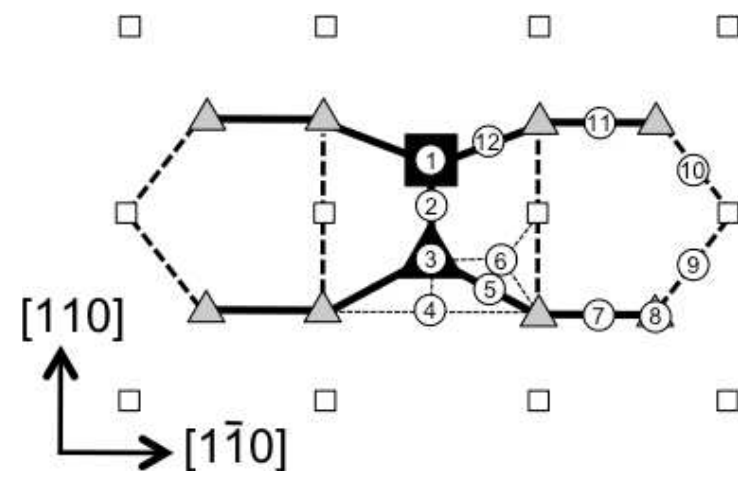

FIG. 1. Tested adsorption sites for oxygen and hydroxyl $(\theta=0.1)$ on the mixed-dimer $\delta(2 \times 4)$ reconstruction of $\mathrm{InP} / \mathrm{GaP}(001)$. Triangles/squares represent $M / \mathrm{P}$ atoms. Larger size and darker shading reflects higher relative atomic position.

constructed surface without the adsorbate; for all other cases, including the $(2 \times 2)$ and $(1 \times 2)$ surfaces, the reference is the dimerized $(1 \times 2)$ surface without the adsorbate. Two different gas adsorbate references were used: (1) $\mathrm{O}_{2}$, and $(2) \mathrm{H}_{2} / \mathrm{H}_{2} \mathrm{O}$. For the projected densities of states, the in-plane k-point mesh density was increased fourfold in each direction. Levels were aligned across different surfaces by matching the average electrostatic potential in the slab center, similar to Ref. ${ }^{46}$. Additional tests using an alignment scheme based on the indium $d$ levels in the slab center gave closely compatible results.

\section{SURFACE STRUCTURE AND ENERGETICS}

\section{A. Oxygen on In/Ga-rich $\operatorname{InP} / \mathrm{GaP}(001)$}

Formation energies $\Delta E$ for stable oxygen adsorption on each of the tested surfaces are reported for both InP and $\mathrm{GaP}$ in Fig. 3. The energies are given with respect to two different gas reference states: (1) $\mathrm{O}_{2}$, and (2) $\mathrm{H}_{2} / \mathrm{H}_{2} \mathrm{O}$. Five local oxygen bond topologies can be identified from the relaxed structures: (1) $M-\mathrm{O}-\mathrm{P}$; (2) $M-$ $\mathrm{O}-M$; (3) a three-way $M_{2}-\mathrm{O}-\mathrm{P}$ bridge; (4) a three-way $M_{2}-\mathrm{O}-M$ bridge; and (5) $\mathrm{P}=\mathrm{O}$, which appears only on the $M-\mathrm{P}$ surface dimer of the mixed-dimer $\delta(2 \times 4)$ reconstruction. Of these, the two-way bridges $M-\mathrm{O}-\mathrm{P}$ and $M-$ $\mathrm{O}-M$ are the most commonly observed and generally the most stable (exceptions are discussed below). The specific location of the adsorbed oxygen has only a minor impact on the energetics of the binding; rather, the stability is primarily determined by the bond topology. Oxygen adsorption is thermodynamically favored with respect to $\mathrm{O}_{2}$ gas (dry atmospheric conditions; left axis in Fig. 3), in agreement with observations of spontaneous surface oxidation of $\operatorname{InP}(001)$ and $\operatorname{GaP}(001)^{8,9,13,26}$. However, only $\mathrm{Ga}-\mathrm{O}-\mathrm{Ga}$ is thermodynamically favored with respect to (a) Oxygen
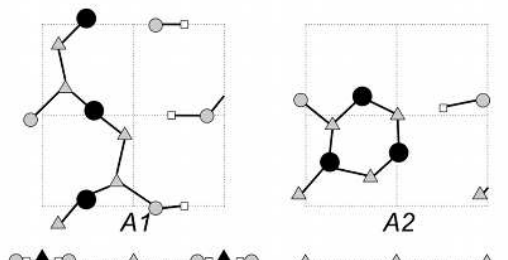

A2
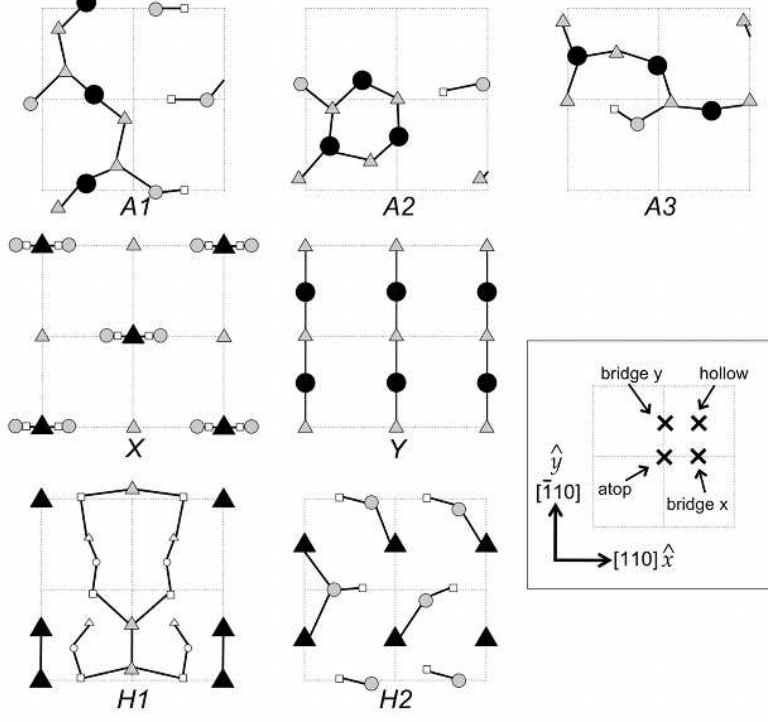

(b) Hydroxyl

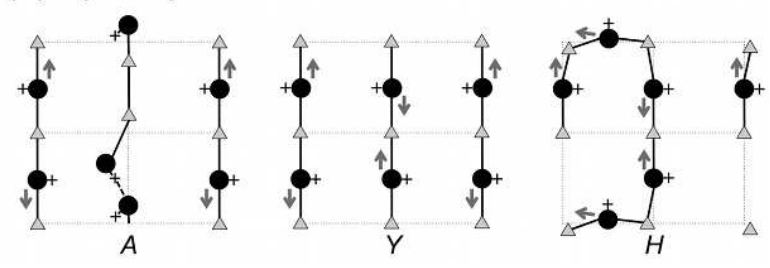

FIG. 2. Schematic representations and designations of relaxed structures for the (a) oxygen-rich and (b) hydroxyl-rich reconstructions generated from the initially unreconstructed $(2 \times 2)$ surface by occupying the high-symmetry sites indicated in the panel inset $(\theta=1)$. Triangles/squares/circles/crosses represent $M / \mathrm{P} / \mathrm{O} / \mathrm{H}$ atoms. Larger size and darker shading reflects higher relative atomic position. Intersections of the dotted square lattice indicate the initial locations of the surface $M$ atoms. For the hydroxylated surfaces in (b), the results are shown for $\operatorname{InP}(001)$; the arrows show the oxygen displacements that generate the corresponding $\mathrm{GaP}(001)$ surface.

$\mathrm{H}_{2} / \mathrm{H}_{2} \mathrm{O}$ (right axis in Fig. 3), which is a better approximation for the operating conditions within a hydrogenevolving photoelectrochemical cell with an aqueous electrolyte. It is worth emphasizing that oxide films grown at ambient temperatures are commonly far from equilibrium $^{24}$. As such, it is appropriate to include in any analysis not only the lowest-energy structure in Fig. 3, but also motifs which represent a somewhat larger range of energies.

The $M-\mathrm{O}-\mathrm{P}$ topology has a similar formation energy for $\mathrm{GaP}$ as for $\mathrm{InP}$, generally within $0.2 \mathrm{eV}$. In the case of InP, In-O-P is also competitive with In-O-In. On the other hand, for $\mathrm{GaP}, \mathrm{Ga}-\mathrm{O}-\mathrm{Ga}$ is clearly preferred over Ga-O-P by more than $0.5 \mathrm{eV}$. This can be traced to the higher strength and covalency of the $\mathrm{Ga}-\mathrm{O}$ bond relative 


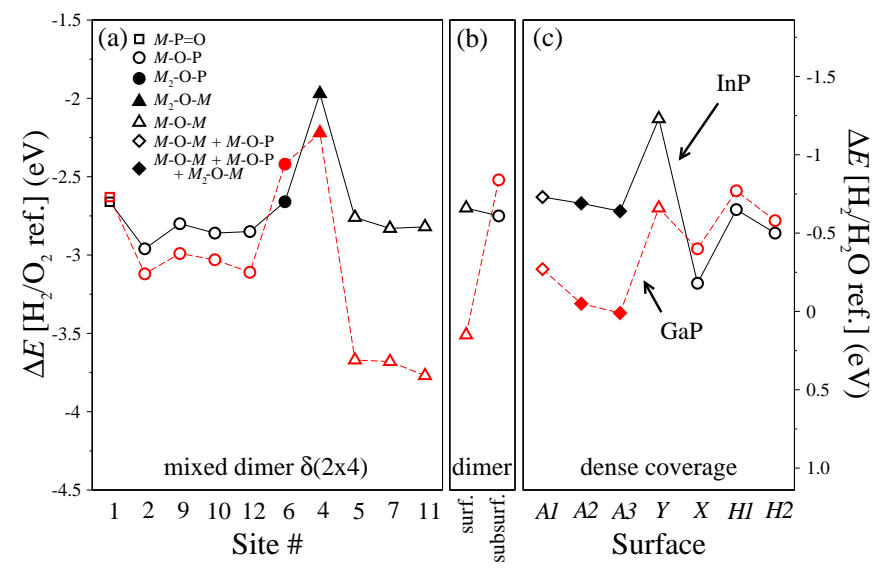

FIG. 3. Formation energies $\Delta E$ (eV per adsorbate) for stable geometries with oxygen adsorbed on (a) the mixed-dimer $\delta(2 \times 4)$ at the sites listed in Fig. $1(\theta=0.1)$; (b) the $(1 \times 2)$ model dimer surface $(\theta=0.5)$; and (c) the $(2 \times 2)$ initially reconstructed surface at the dense limit $(\theta=1)$ to generate the surfaces in Fig. 2a. Black solid (red broken) lines are for $\mathrm{InP}(\mathrm{GaP})$. Values are given with respect to $\mathrm{H}_{2} / \mathrm{O}_{2}$ (left axis) and $\mathrm{H}_{2} / \mathrm{H}_{2} \mathrm{O}$ (right axis) gas references (see Methods for details). The symbols correspond to the different constituent bond topologies indicated in the legend. In (a), adsorption on Site \#4 of the mixed-dimer surface pushes the $M-\mathrm{P}$ dimer along [110] to equalize the three bond lengths, resulting in higher $E_{\text {ads }}$; other surfaces retain their basic structure.

to the In-O bond in these structures, which matches the trend for the $M-\mathrm{P}$ bonds in the corresponding bulk materials ${ }^{47}$. Accordingly, we observe lower formation energies for oxygen-decorated $\mathrm{GaP}$ over $\mathrm{InP}$ at nearly all equivalent binding sites that contain $M-\mathrm{O}$ bonds, with the difference generally proportional to the $M-\mathrm{O}$ concentration (for instance, among the dense-coverage surfaces, the largest differences are found for $A 1, A 2, A 3$, and $Y$ ). The same scenario is reflected in the bond lengths in InP and $\mathrm{GaP}$, which differ on average by $\sim 0.35 \AA$ for $\mathrm{Ga}-\mathrm{O}$ and $\mathrm{In}-\mathrm{O}$. One might also expect the $\mathrm{P}-\mathrm{O}$ bond strength to be affected by the identity of the metal species to which the oxygen is bound. However, this does not seem to be the case, as $\mathrm{O}-\mathrm{P}$ bond lengths are essentially identical for the In-O-P and $\mathrm{Ga}-\mathrm{O}-\mathrm{P}$ topologies. This hints at the intrinsically local nature of the $M-\mathrm{O}-\mathrm{P}$ bond chemistry.

Collective examination of the various surface morphologies suggests that whereas the $M-\mathrm{O}-M$ motif in $\mathrm{InP} / \mathrm{GaP}(001)$ can exist only at the surface, where neighboring In or Ga atoms are exposed to one another, the $M-\mathrm{O}-\mathrm{P}$ motif can substitute for any existing $M-\mathrm{P}$ bond as long as the induced lattice strain can be accommodated. Based on results for the mixed-dimer $\delta(2 \times 4)$, this strain represents $35-40 \%$ expansion with respect to the bulk $M-\mathrm{P}$ distance. The mixed-dimer $(2 \times 4)$ can feature $M-\mathrm{O}-\mathrm{P}$ as a surface motif, since a surface phosphorus atom is present. However, the $(1 \times 2)$ dimer and initially unreconstructed dense-coverage surfaces in (Figs. 2a, 3b, and 3c) contain no surface phosphorus. Instead, the expression of phosphorus-containing motifs on these reconstructions do not derive from surface-layer oxygen but rather from oxygen incorporation into the subsurface layer. Some structures (e.g., $X$ ) demonstrate significant surface corrugation as a consequence. Three different depths of $M-\mathrm{O}-\mathrm{P}$ oxygen penetration are represented in Fig. 2. The $X$ structure pushes some In/Ga atoms into a new surface layer, bridged by oxygen atoms to a phosphorus subsurface. The H2 structure features oxygens directly below the primary $M$-rich surface layer. In $H 1$, the oxygens sit below the subsurface phosphorus atoms. Notably, there is an inverse correlation between oxygen depth in these three surfaces and their corresponding $\Delta E$.

To gain a more direct assessment of the thermodynamics of oxygen incorporation into the subsurface layers, we also calculated the energetic cost of inserting oxygen into $M-\mathrm{P}$ bonds beneath the surface of the mixed-dimer $(2 \times 4)$ reconstruction. For the surface and first three subsurface layers, those calculations showed $\mathrm{O}_{2}$-referenced formation energies of $-2.98 /-3.13, \quad-2.49 /-2.59$, $-2.40 /-2.26,-2.26 /-2.26 \mathrm{eV}$ for $\operatorname{InP}(001) / \mathrm{GaP}(001)$. Again, we observe an inverse correlation between the depth and corresponding $\Delta E$ for the first few layers, although the values appear to saturate quickly. Note that these values also favor oxygen incorporation in the bulk; however, they do not account for potentially unfavorable kinetics at ambient temperatures.

Two of the dense-coverage surfaces in Figs. 2a and 3c merit further mention. First, the $X$ configuration has unexpectedly low $\Delta E$, particularly for InP. This is because the resulting surface reconstruction features formation of stable $\mathrm{P}-\mathrm{P}$ dimers. Second, the $Y$ configuration has a higher-than-expected $\Delta E$, despite being highly ordered and featuring only $M-\mathrm{O}-M$ bridge motifs, which the other samples show to be quite stable. This seeming inconsistency turns out to be connected to the lattice strain induced by symmetry constraints from simultaneous occupation of neighboring oxygen bridge sites. This is seen by comparing $Y$ to the bridge y configuration in the $(1 \times 2)$ dimer supercell, which has a lower surface adsorbate concentration of $\theta=0.5$. Since the $\theta=1 \quad Y$ surface forces the surface $M-M$ distance to match the bulk distance, the change in this quantity upon lowering the surface adsorbate concentration acts as an indicator of the accompanying strain relaxation. For surface oxygen, the strain relaxation is substantial, with a $20-23 \%$ decrease in the $M-M$ distance, and a corresponding change in the $M-\mathrm{O}-M$ bond angle from $157-158^{\circ}$ to $113^{\circ}$. As discussed below, this strain also profoundly impacts the accompanying local electronic structure.

\section{B. Hydroxyl on In/Ga-rich InP/GaP(001)}

Oxygen adsorption could also occur via surface hydroxylation, particularly in a moisture-rich environment. 
As such, the adsorption calculations were repeated for hydroxyl-decorated InP/GaP(001) surfaces. Compared to the oxygen-decorated case, the hydroxylated surface shows a simpler configurational phase space with fewer dominant local bond topologies.

Figure 4 shows the formation energies for stable adsorption of hydroxyl on our tested surfaces. We find three local oxygen bond topologies: a $\mathrm{P}-\mathrm{OH}$ atop bond; a $M-$ $\mathrm{OH}$ atop bond; and a $M-[\mathrm{OH}]-M$ bridge. For the mixeddimer $(2 \times 4)$, the $M-[\mathrm{OH}]-M$ bridge shows a strong preference over the atop configurations, being thermodynamically favored by $0.6-0.7 \mathrm{eV}$. Interestingly, this is not consistent across the three categories of surfaces, indicating some dependence of the adsorption energy on the broader environment. For instance, the ordering is reversed for the $(1 \times 2)$ dimer surface. This is because the displacement constraints on the mixed-dimer $(2 \times 4)$ reconstruction that arise from surface atoms bound to the $M-M$ dimer are not present for the dimerized $(1 \times 2)$ reconstruction. This permits energy-lowering surface buckling for the $(1 \times 2)$ reconstruction but not for the mixed-dimer $(2 \times 4)$, as detailed further below.

As we saw for the oxygen-adsorbed surface, $\operatorname{GaP}(001)$ consistently binds hydroxyl more strongly than $\operatorname{InP}(001)$ whenever a $M-\mathrm{OH}$ bond is present, owing to the relative strength of the $\mathrm{Ga}-\mathrm{O}$ bond over the $\mathrm{In}-\mathrm{O}$ bond. However, unlike the oxygen case, the strength of binding does not seem to scale with the number of $M-\mathrm{OH}$ bonds, since the bridge and atop topologies exhibit nearly identical behavior. Much like the $\mathrm{P}=\mathrm{O}$ bond in Fig. 3a, the $\mathrm{P}-$ $\mathrm{OH}$ bond in Fig. 4a carries the same energy for both GaP and InP, confirming the validity of a local representation for this interaction.

Comparing Figs. 3 and 4, we find that hydroxyl adsorption on mixed-dimer $\delta(2 \times 4)$ is energetically favored with respect to oxygen adsorption, assuming sufficient proton concentration. As such, the exposed outermost surface layer of $\mathrm{GaP} / \operatorname{InP}(001)$ is likely to hydroxylate spontaneously. This agrees with recent calculations showing preferred hydroxylation on exposed (001) surfaces of InP nanowires, for instance ${ }^{48}$. It is worth noting that the $M-$ $[\mathrm{OH}]-\mathrm{P}$ configuration is absent from Fig. 4, since we were unable to stabilize it; hydroxyl groups are thus likely to exist only as surface species except at very high concentrations.

Hydroxyl adsorption on the initially unreconstructed In/Ga-rich $(2 \times 2) \operatorname{InP} / \mathrm{GaP}(001)$ surface slab generated slightly different geometries for $\mathrm{GaP}(001)$ and $\mathrm{InP}(001)$, as shown in Fig. 2b. The structural differences between the hydroxylated $\operatorname{InP}(001)$ and $\mathrm{GaP}(001)$ surfaces can be traced to a symmetry-breaking distortion that occurs on $\mathrm{GaP}$ when neighboring bridge sites are occupied with hydroxyl groups. The $-[\mathrm{OH}]-$ bridge is not evenly shared between $M$ atoms as it is for InP, but rather is weakly bound to one Ga atom and strongly to the other (e.g., for the $Y$ configuration, the bond distances are 1.98 and $2.31 \AA$ ). In addition, the strong-binding Ga atom prefers to host two shorter $\mathrm{Ga}-[\mathrm{OH}]$ bonds, leaving the weak-

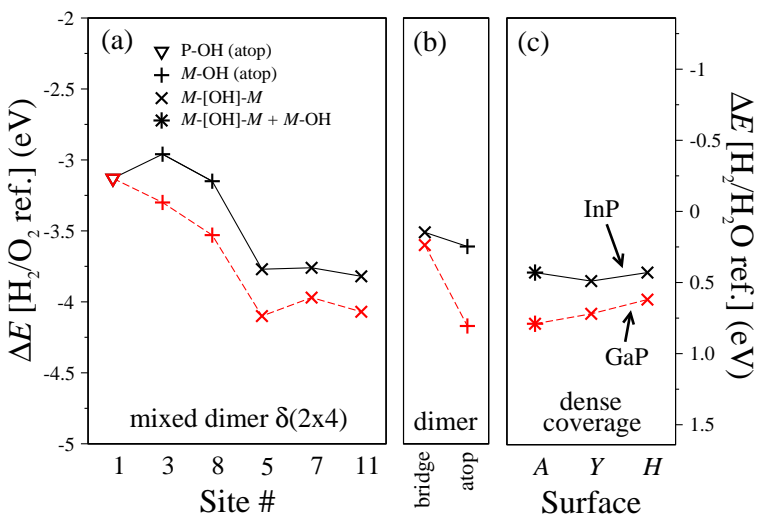

FIG. 4. Formation energies $\Delta E$ (eV per adsorbate) for stable geometries with hydroxyl adsorbed on (a) the mixed-dimer $\delta(2 \times 4)$ at the sites listed in Fig. $1(\theta=0.1)$; $(\mathrm{b})$ the $(1 \times 2)$ model dimer surface $(\theta=0.5)$; and $(\mathrm{c})$ the $(2 \times 2)$ initially reconstructed surface at the dense limit $(\theta=1)$ to generate the surfaces in Fig. 2b. Black solid lines are InP; red broken lines are $\mathrm{GaP}$. Values are given with respect to $\mathrm{H}_{2} / \mathrm{O}_{2}$ (left axis) and $\mathrm{H}_{2} / \mathrm{H}_{2} \mathrm{O}$ (right axis) gas references (see Methods for details). The symbols correspond to the different constituent bond topologies indicated in the legend.

binding $\mathrm{Ga}$ atom with both longer $\mathrm{Ga} \cdots[\mathrm{OH}]$ bonds. The result is an alternating $[\mathrm{OH}] \cdots \mathrm{Ga} \cdots[\mathrm{OH}]-\mathrm{Ga}-[\mathrm{OH}]$ complex.

There is a second structural difference between GaP and $\mathrm{InP}$ that is seen only for the $\theta=0.5-[\mathrm{OH}]-$ bridge on the $(1 \times 2)$ dimer surface. Here, GaP displays significant surface buckling that does not occur for InP, with one Ga atom rising out of the surface plane, $0.57 \AA$ above its counterpart. Similar buckling is commonly observed in other semiconductor surfaces ${ }^{49}$, and has been proposed as a surface structural motif in $\mathrm{GaInP}_{2}{ }^{45,50}$. Notably, this same type of buckling is observed on the $(1 \times 2)$ dimer surface of both $\mathrm{GaP}$ and $\mathrm{InP}$ upon adsorption of a single atop $\mathrm{OH}$ on the $M-M$ dimer, although the out-of-plane displacement of the surface $M$ atom is somewhat smaller in this case $(0.30 \AA$ for $\mathrm{GaP} ; 0.23 \AA$ for $\mathrm{InP})$. In each instance, the buckling results from $s p^{2}$ hybridization of one of the $M$ edge atoms and transfer of charge to the other $M$ atom, which acquires atomic-like $p$ orbitals. The charge redistribution also breaks the symmetry in the two sets of $\mathrm{P}-M-\mathrm{O}$ angles spanned by the edge $M$ atoms and their subsurface $\mathrm{P}$ atoms (the angles become $94^{\circ}$ and $117^{\circ}$ for $\mathrm{Ga}-[\mathrm{OH}]-\mathrm{Ga} ; 95^{\circ}$ and $116^{\circ}$ for $\mathrm{Ga}-\mathrm{Ga}-[\mathrm{OH}]$; and $95^{\circ}$ and $114^{\circ}$ for $\left.\mathrm{In}-\mathrm{In}-[\mathrm{OH}]\right)$. An additional possible consequence of this buckling is to expose a neighboring subsurface phosphorus atom to easier adatom attack.

The $H$ configuration in Figs. $2 \mathrm{~b}$ and $4 \mathrm{c}$ features $M-$ $[\mathrm{OH}]-M$ bridge hydroxyls oriented along [110] $(\hat{x})$, in contrast with the oxygen-decorated case, where $M-\mathrm{O}-$ $M$ bridges were found oriented only along [110] $(\hat{y})$. The reason for this is that unlike oxygen, insertion of hydroxyl in a bridge changes the bulk equilibrium $M-M$ bond dis- 


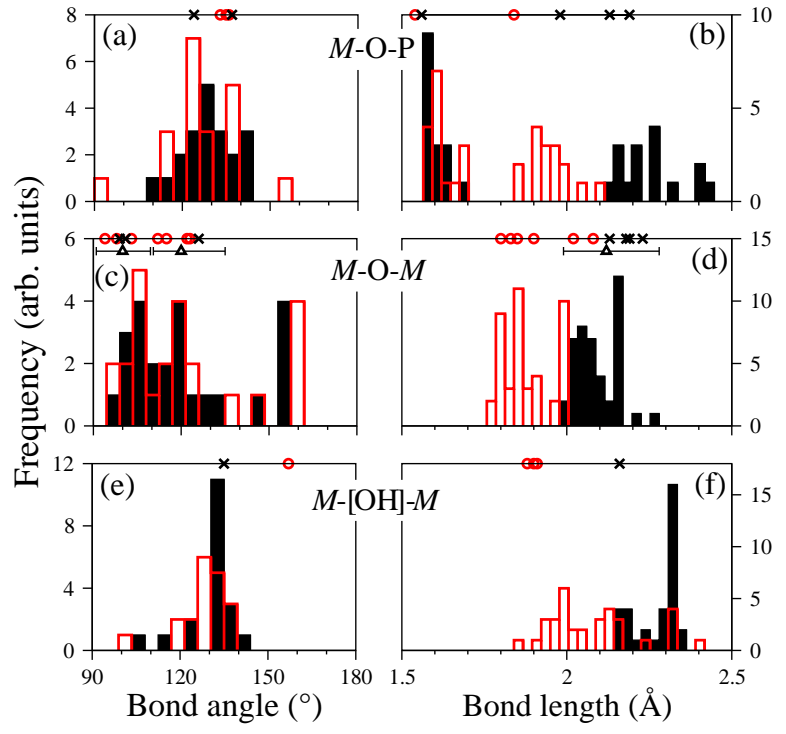

FIG. 5. Histograms of bond distances $(M-\mathrm{O}, \mathrm{O}-\mathrm{P})$ and bond angles $(M-\mathrm{O}-M, M-\mathrm{O}-\mathrm{P})$ for the (a-b) $M-\mathrm{O}-\mathrm{P}$, (c-d) $M-$ $\mathrm{O}-M$, and (e-f) $M-[\mathrm{OH}]-M$ bond topologies, sampled across all tested $\operatorname{InP}(001)$ and $\mathrm{GaP}(001)$ surfaces. Solid black bars are $\mathrm{InP}$ values; hollow red bars are $\mathrm{GaP}$ values. In (b), values below $1.8 \AA$ represent $\mathrm{O}-\mathrm{P}$ bonds. Experimental values for bulk orthorhombic $\mathrm{InPO}_{4}$ (Ref. ${ }^{51}$ ), bixbyite $\mathrm{In}_{2} \mathrm{O}_{3}$ (Ref. ${ }^{52}$ ), and $\mathrm{In}(\mathrm{OH})_{3}$ (Ref. $^{53}$ ) are shown as black $\times$ in panels $\mathrm{a}-\mathrm{b}$, $\mathrm{c}-\mathrm{d}$, and e-f, respectively; quartz-type $\mathrm{GaPO}_{4}$ (Ref. $\left.{ }^{54}\right), \beta$ $\mathrm{Ga}_{2} \mathrm{O}_{3}$ (Ref. $^{55}$ ), and $\mathrm{Ga}(\mathrm{OH})_{3}$ (Ref. $^{56}$ ) are shown as red circles. In panels c-d, distributions for amorphous $\operatorname{In}_{2} \mathrm{O}_{3}$ are also reported (black triangles, Ref. ${ }^{57}$ ), with attached error bars representing full-width half maxima.

tance only slightly, easing the strain requirement for $\hat{x}$ oriented bridges. For instance, the $M-M$ distance in the $(1 \times 2)$ dimer with a hydroxyl bridge is changed from the bulk distance by only $1 \%$ for $\mathrm{InP}$ and $4 \%$ for $\mathrm{GaP}$.

The morphology and energetics of the $A$ configuration in Figs. 2b and 4c hints at the importance of hydrogen bonding for dense hydroxyl coverages. Here, hydrogen bonding between neighboring atop-site hydroxyl groups stabilizes the surface significantly, reconciling its low formation energy with the thermodynamic preference for the $M-[\mathrm{OH}]-M$ bridge over the atop $M-\mathrm{OH}$ on the mixed-dimer $\delta(2 \times 4)$ reconstruction in Fig. 2a. In addition, since the formation energies of the three configurations in Fig. 4c are very close and the corresponding geometries differ only by a few simple bond rearrangements, one may expect dynamical interconversion between them at the interface with liquid water, whose hydrogen bonding can compete directly with $M-\mathrm{OH} \cdots \mathrm{OH}-M$ and $M-$ $[\mathrm{OH}]-M$ bond formation to lower kinetic barriers.

\section{LOCAL BOND GEOMETRIES}

Having cast the complex morphologies of oxygen- and hydroxyl-rich surfaces in terms of local bond topologies, we use our generated surfaces to isolate commonalities in the associated bond geometries. Structural features can then be compared to measurements in bulk oxides and hydroxides to assess the validity and universality of our models, and to provide potential insight into the local bond configurations that can lead to initial nucleation of native oxide and hydroxide films. In doing so, we find compelling structural parallels between our results and the reported corrosion byproducts of InP and GaP photoelectrodes, namely, $M_{2} \mathrm{O}_{3}$-type oxides and $M \mathrm{PO}_{4}$-type phosphates, as well as $M(\mathrm{OH})_{3}$ and $\mathrm{P}_{2} \mathrm{O}_{5} \cdot{ }^{9,10,24-30}$. Similar products are reported for the $\mathrm{In}_{0.5} \mathrm{Ga}_{0.5} \mathrm{P}$ alloy. ${ }^{7,58}$

Fig. 5 shows histograms of key bond angles and bond distances in the $M-\mathrm{O}-M, M-\mathrm{O}-\mathrm{P}$, and $M-[\mathrm{OH}]-M$ bond topologies, compiled from the entire set of InP and GaP samples we investigated. Although one should be careful drawing quantitative conclusions, certain qualitative features can nevertheless be extracted for comparison among bond topologies and with experimentally reported values. Results for InP and GaP are qualitatively similar, with the exception of the different equilibrium $M-$ $\mathrm{O}$ bond distances (in contrast, the $\mathrm{O}-\mathrm{P}$ bond lengths in Fig. 5b are the same for the two). The distribution of $M-$ $\mathrm{O}$ bond distances for $M-[\mathrm{OH}]-M$ is also wider for $\mathrm{GaP}$ than for InP, which derives from the symmetry-breaking distortion already described.

Key differences are evident in the distributions of the bond distances and angles when comparing $M-\mathrm{O}-\mathrm{P}$ and $M-\mathrm{O}-M$ topologies. For instance, the range of bond angles for $M-\mathrm{O}-M$ (Fig. 5c) is generally broader than for $M-\mathrm{O}-\mathrm{P}$ (Fig. 5a), indicating the greater covalency of the $\mathrm{O}-\mathrm{P}$ bond. Nevertheless, the $M-\mathrm{O}-M$ distribution has more internal fine structure. The $M-\mathrm{O}-\mathrm{P}$ angles show possibly two peaks appearing around 125 and 135 , whereas the $M-\mathrm{O}-M$ angles have what appears to be three distinct peaks around $100^{\circ}, 120^{\circ}$, and $160^{\circ}$. Similarly, the distributions of $M-\mathrm{O}$ bond distances show discernible fine structure for $M-\mathrm{O}-M$ but not for $M-\mathrm{O}-\mathrm{P}$, with two clear peaks visible for InP and possibly three for $\mathrm{GaP}$.

The fine structure in our $M-\mathrm{O}-M$ distributions correlates to different local configurations or hybridizations. In Fig. 5c, the $M-\mathrm{O}-M$ bond angle peak near $160^{\circ}$ represents a bridge oxide that is strained by symmetry constraint, e.g., $Y(\theta=1)$. This bond angle is not found in the bulk oxides, probably because of the associated strain energy. However, the other two peaks (around $100^{\circ}$ and $120^{\circ}$ ) are common across a variety of tested surfaces, and closely match reported values for the common polymorphs $\beta-\mathrm{Ga}_{2} \mathrm{O}_{3}$ and bixbyite $\mathrm{In}_{2} \mathrm{O}_{3}$, as well as In-O-In angular distributions in amorphous $\mathrm{In}_{2} \mathrm{O}_{3}{ }^{52,55,57}$. Similarly, the $\mathrm{Ga}-\mathrm{O}$ bond distances in Fig. 5 d show peak splitting reminiscent of the different intrinsic oxygen-bridge geometries of $\mathrm{GaO}_{4} / \mathrm{GaO}_{6}$ in $\beta-\mathrm{Ga}_{2} \mathrm{O}_{3}{ }^{55}$, and the longer- 
distance In-O bonds are close to the reported values for bixbyite $\mathrm{In}_{2} \mathrm{O}_{3} \cdot{ }^{52}$ In addition, the shorter-distance In-O distribution resembles the shape reported for amorphous $\mathrm{In}_{2} \mathrm{O}_{3} \cdot{ }^{57}$

The internal structure of the $M-\mathrm{O}-\mathrm{P}$ bond angle histogram (Fig. 5a) is somewhat less resolvable than for $M-$ O-M (Fig. 5d); nevertheless, the two apparent peak locations appear to match discrete values for hydrous and anhydrous $\mathrm{InPO}_{4}{ }^{51,59,60}$. The $\sim 135^{\circ}$ peak also matches quartz-type $\mathrm{GaPO}_{4}{ }^{54,61}$. Although the smaller $\mathrm{Ga}-\mathrm{O}-\mathrm{P}$ angle $\left(\sim 120^{\circ}\right)$ is not found at room temperature, the fact that $\mathrm{GaPO}_{4}$ is isomorphic with $\mathrm{InPO}_{4}$ at high pressure suggests this type of bond could manifest under nonequilbrium growth conditions ${ }^{62}$. It is worth noting that calculated $M-\mathrm{O}$ bond lengths for the $M-\mathrm{O}-\mathrm{P}$ topology (Fig. 5b) are noticeably shorter than in bulk $M \mathrm{PO}_{4}$. This is probably indicative of the additional geometric constraint placed on our system by the underlying $\mathrm{InP} / \mathrm{GaP}$ lattice.

In contrast with the oxides, the angular distributions for the $M-[\mathrm{OH}]-M$ bridge (Fig. 5e) do not exhibit additional peak structure, but are normally distributed about an angle of $130-135^{\circ}$. This suggests that in our hydroxide simulations, the oxygen complexes do not have multiple preferred hybridizations. The peak is a good match to bulk $\operatorname{In}(\mathrm{OH})_{3}$ but differs significantly from $\mathrm{Ga}(\mathrm{OH})_{3}$, which adopts a different oxygen environment that is apparently not captured by our calculations. In addition, the calculated $M-\mathrm{O}$ bond lengths for $M-[\mathrm{OH}]-$ $M$ (Fig. 5f) are shorter than in bulk $\mathrm{M}(\mathrm{OH})_{3}$, much as we saw for the $M-\mathrm{O}-\mathrm{P}$ topology in Fig. 5b. Note the wide distribution of $\mathrm{Ga}-\mathrm{O}$ bond lengths in Fig. 5f, which is a consequence of the symmetry-breaking distortion described earlier.

\section{SURFACE ELECTRONIC STRUCTURE}

To determine the effect of adsorption on the surface electronic structure, we calculated projected densities of states (PDOS) for atoms in the local oxygen bond environments of several of our tested $\mathrm{InP} / \mathrm{GaP}(001)$ surfaces. For brevity, we only present results for $\operatorname{InP}(001)$ here. Select results for $\mathrm{GaP}(001)$ are provided in the supplementary information ${ }^{63}$; it should be noted that there is generally qualitative agreement between the two species. The PDOS for select distinct bond topologies are shown in Figs. 6-9. Each of the local bond topologies demonstrates unique electronic signatures that should permit identification using available experimental techniques.

For comparison with the bulk and surface band edges, the Figures also show the density of states of bulk InP, as well as the total PDOS of the surface atoms in the clean mixed-dimer $(2 \times 4)$ and the clean $(1 \times 2)$ dimer surfaces (Figs. 6a, 7a, 9a). The presence of the clean surfaces changes the character and position of the band edges with respect to the bulk, particularly for the conduction band, resulting in a shrinking of the gap. The $(1 \times 2)$ dimer sur- face also shows a localized donor level due to dangling bond states, which disappears upon further surface adsorption.

\section{A. Oxygen on In-rich $\operatorname{InP}(001)$}

Figure 6 displays the PDOS near the Fermi level for the $\mathrm{In}, \mathrm{O}$, and $\mathrm{P}$ atoms in a sampling of topologically unique In-O-P topologies. The valence signatures of the surfaces are similar, despite significant differences in the surface coordination and atomic arrangement. In several cases, the oxygen $2 p$ states hybridize strongly with In and $\mathrm{P}$ neighbors to form an $\mathrm{In} / \mathrm{O} / \mathrm{P}$ peak at around $-6.5 \mathrm{eV}$ representing a three-center bond. This is seen for the In-O-P topologies on the mixed-dimer surface, as well as the $X$ configuration (Fig. $6 \mathrm{~b}-\mathrm{d}, \mathrm{f}$ ), but does not appear in the clean surface (Fig. 6a). A second set of oxygen peaks with a degree of In and $\mathrm{P}$ hybrid character emerges in the vicinity of $-4.0 \mathrm{eV}$. Notably, peaks in locations similar to the $-4.0 \mathrm{eV}$ and $-6.5 \mathrm{eV}$ peaks recorded in Fig. 6 were also identified in photoemission spectra of native oxide nucleation on $\operatorname{InP}(110)^{25,64}$. The amplitude of these peaks was found to directly correlate with oxygen exposure. Our results suggest that the observed oxide is $\mathrm{InPO}_{4}$-like in nature, consisting of $\mathrm{In}-\mathrm{O}-\mathrm{P}$ local bond topologies. This is consistent with the structure of $\operatorname{InP}(110)$, which consists of exposed In-P surface dimers that can act as oxygen insertion sites. On the mixed-dimer $\delta(2 \times 4)$ surface (Fig. $6 \mathrm{~b}-\mathrm{e})$, there is also significant enhancement of the peak at the valence-band maximum, which appears regardless of absorption site. If localized both energetically and spatially, these states may translate to reduced hole mobility near the defect site, affecting photoelectrode performance. A combined experiment-theory effort is currently underway to confirm these findings.

The character of the primarily In-derived conduction band is also qualitatively similar across the tested samples. The band edges show little movement with respect to the clean surfaces in Fig. 6a, expressing only minor valence-band shifts towards the gap for the $A 1$ and subsurface-oxygen $(1 \times 2)$ dimer surfaces (Fig. 6g\&h).

The PDOS for several of the In-O-In topologies is shown in Fig. 7. In contrast to the In-O-P topology, the surfaces in Fig. 7 all feature significant oxygen $2 p$ peaks that are localized within the first few $\mathrm{eV}$ below the Fermi level that contain little In character. However, the location of these peaks is highly variable across tested surfaces. For some, such as the $Y$ structure with $\theta=1$ (Fig. 7e) and the double-edge bound $\mathrm{H}-\mathrm{In}-\mathrm{O}-\mathrm{In}-\mathrm{H}$ bridge (Fig. $7 \mathrm{~h}$ ), the peak arises near the valence band maximum. In this case, the PDOS acts as a signature of hole carrier trapping, which we explore in detail below. For other surfaces, the peak is less localized and deeper in the valence by as much as $3 \mathrm{eV}$.

The position of the itinerant oxygen peaks in the In-OIn topologies turns out to be closely coupled to the In-O 


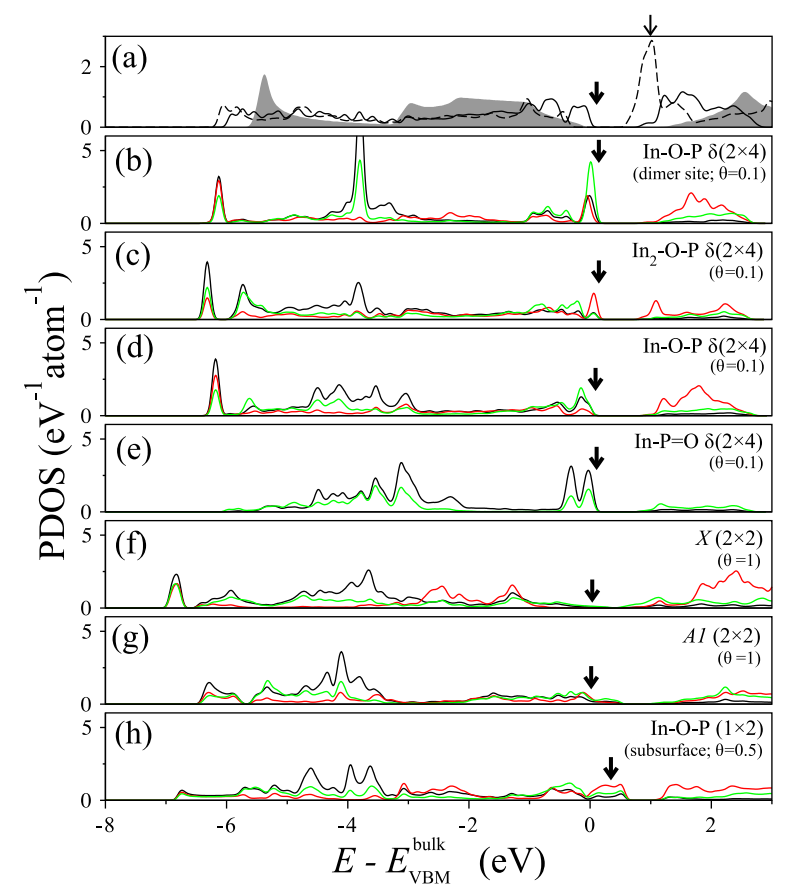

FIG. 6. (Color online) PDOS for surface atoms of some local $\mathrm{InP}(001)$ bond topologies featuring $\mathrm{O}-\mathrm{P}$ bonds. Black/red/green curves represent $\mathrm{O} / \mathrm{In} / \mathrm{P}$ densities of states. Highest occupied surface states are shown as arrows. Panel (a) shows the bulk density of states (filled region) alongside the surface PDOS for the clean mixed-dimer $\delta(2 \times 4)$ (solid line; thick arrow) and the clean $(1 \times 2)$ dimer (dashed line; thin arrow) surfaces. The remaining configurations represent: (be) sites \#2, \#6, \#9, and \#1 on the mixed-dimer $\delta(2 \times 4)$ (see Fig. 1); (f-g) the $X$ and $A 1$ dense-coverage surfaces (see Fig. 2a); and $(\mathrm{h})$ the $(1 \times 2)$ dimer lattice with a subsurface oxygen inserted between In and P. Energies are referenced to the valence-band maximum of bulk InP.

bond distance and In-O-In bond angle, which are listed alongside the PDOS for each of the structures in Fig. 7. Larger angles and longer bond distances tend to drive the oxygen states towards the valence band maximum, likely because this weakens the covalent bonding character in the mixed ionic-covalent In- $\mathrm{O}$ bond, making the oxygen states more atomic-like. The shift is accompanied by an increase in the localization and a decrease in the indium hybrid character of the peak. The link between In-O-In bond geometry and the location of the oxygen $2 p$ valence peak is explored more fully in Fig. 8, for which the In-In distance in the $(1 \times 2)$ dimer with an In-O-In bridge was fixed at different values across the bridge. Remaining atoms were allowed to relax under the constraint. Although the effect of compression on the PDOS is slight, possibly because the In-O bond distance does not change appreciably, expansion sharply shifts the oxygen $2 p$ peak towards the valence band maximum. For $20 \%$ expansion (Fig. 8e), which corresponds approximately to the bulk In-In distance, peak locations are similar those found for the $Y$ oxide with $\theta=1$ (Fig. 7a).

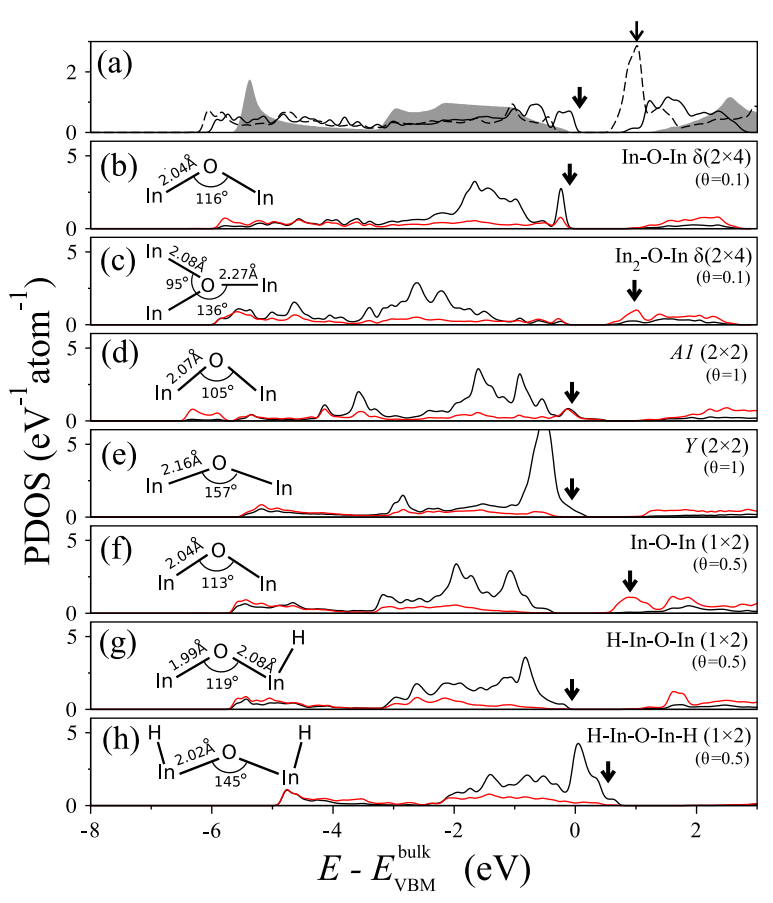

FIG. 7. (Color online) PDOS for surface atoms of some local $\mathrm{InP}(001)$ bond topologies featuring In-O-In bonds. Black/red curves represent $\mathrm{O} / \mathrm{In}$ densities of states. Highest occupied surface states are shown as arrows. See caption of Fig. 6 for an explanation of (a). The remaining configurations represent: (b-c) sites \#5 and \#4 on the mixed-dimer $\delta(2 \times 4)$ (see Fig. 1$)$; (d-e) the $A 1$ and $Y$ dense-coverage surfaces (see Fig. $2 \mathrm{a})$; and $(\mathrm{f}-\mathrm{h})$ the $(1 \times 2)$ dimer lattice with a surface bridge oxygen and with no atop $\mathrm{H}$, with one atop $\mathrm{H}$, and with two atop $\mathrm{H}$, respectively. Energies are referenced to the valence-band maximum of bulk InP.

Altering the chemical binding environment of the endpoint indium atoms can also change the In-O-In bond geometry and affect the location and character of the oxygen $2 p$ valence peaks. To see this, we explored changing the In coordination by binding an atop $-\mathrm{H}$ to one or both In edges of the $(1 \times 2)$ surface with bridge oxygen (Figs. $7 \mathrm{f}-\mathrm{h}$ ). The effect of the termination of the dangling edge bonds is to drive the oxygen valence peaks towards the Fermi energy and to make the corresponding states more localized and atomic-like. Binding to both edge atoms strains the In-O-In bond more than binding to one of the edge atoms, which is reflected in higher oxygen $2 p$ energies.

The In-O-In conduction states (Fig. 7) are more dispersive than for In-O-P (Fig. 6), as indicated by the shallower slope in the PDOS. Our results indicate that the position of the indium-derived surface conduction band edges in the In-O-In PDOS is largely determined by the availability of indium binding sites. More specifically, the existence of unsaturated dangling bonds on the bridgeterminating In atoms tends to systematically lower the band edge with respect to the bulk. For instance, this 


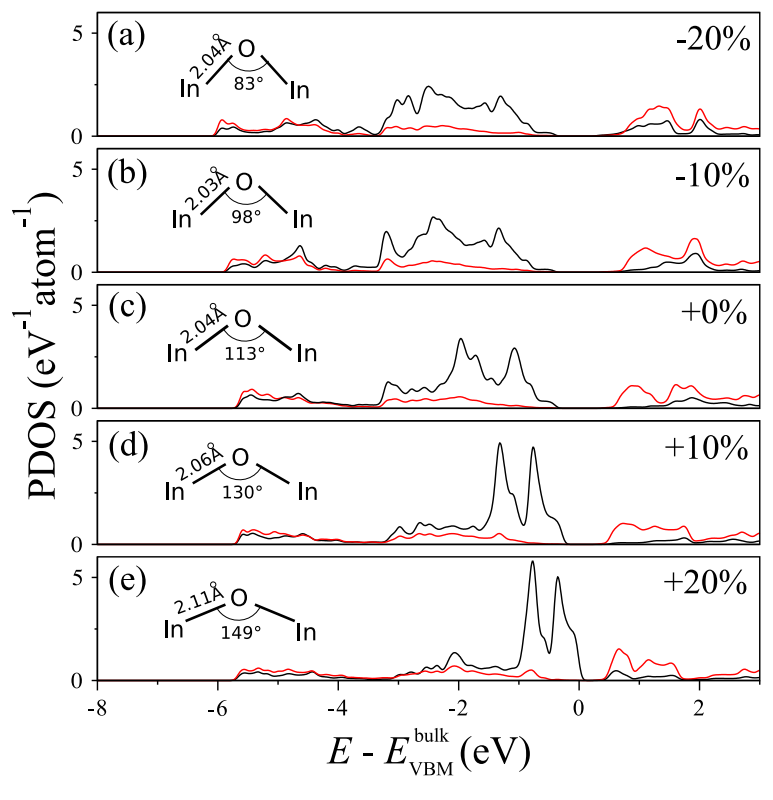

FIG. 8. (Color online) PDOS for surface In and $\mathrm{O}$ atoms of $\mathrm{InP}(001)$ involved in a bridge y $\mathrm{In}-\mathrm{O}-\mathrm{In}$ bond configuration for $\theta=0.5$ under various levels of strain. With reference to the equilibrium In-In distance, the panels represent: (a) $20 \%$ compression; (b) 10\% compression; (c) the equilibrium distance; (c) 10\% tension; and (d) 20\% tension. Black/red curves represent $\mathrm{O} / \mathrm{In}$ densities of states. Energies are referenced to the valence-band maximum of bulk InP.

conduction-band shift is manifest in comparing the band edge of the $(1 \times 2)$ oxygen bridge (Fig. $7 f$ ), which has dangling bonds, to the same structure with single-edge (Fig. $7 \mathrm{~g}$ ) or double-edge (Fig. $7 \mathrm{~h}$ ) binding of atop $-\mathrm{H}$. Similarly, the $Y$ configuration $(\theta=1$; Fig. 7 e) differs from the $(1 \times 2)$ oxygen bridge (Fig. $7 \mathrm{f})$ in that it has no available edge binding sites, and consequently features a wider gap. Depending on the position of the Fermi level, the conduction-band edge shift can lead to the creation of donor-like states in the gap, as seen in Fig. 7c\&f. Notably, this same phenomenon is observed for the clean $(1 \times 2)$ dimer surface (dashed line in Fig. 7 a; note the difference in scale), which also has dangling bonds associated with the In edge atoms.

\section{B. Hydroxyl on In-rich $\operatorname{InP(001)}$}

The PDOS for surface atoms in In-OH (In-In-OH and $\mathrm{P}-\mathrm{In}-\mathrm{OH}), \mathrm{P}-\mathrm{OH}(\mathrm{In}-\mathrm{P}-\mathrm{OH})$, and several $\mathrm{In}-[\mathrm{OH}]-\mathrm{In}$ bridge structures are shown in Fig. 9. Unlike the In-O-In bridge, the valence band edge is not appreciably affected by the presence of oxygen. Similarly, when one or two atop hydrogen atoms are bound to the edge In atoms of the $(1 \times 2)$ In- $-[\mathrm{OH}]-\mathrm{In}$ bridge (Fig. $9 \mathrm{f}-\mathrm{h})$, there is no shift in the primary valence peaks or valence-band edges, in contrast to what we saw for the In-O-In bridge in Fig. 7fh. As such, surface hydroxylation of $\operatorname{InP}(001)$ is unlikely to significantly impact hole mobility. Nevertheless, the position of the conduction band edge is determined by the same rules as for In-O-In, with unsaturated bonds closing the gap. For example, in Fig. $9 \mathrm{f}-\mathrm{h}$, the location of the donor-like state shifts from a deep donor (Fig. 9f) to a shallow donor (Fig. 9g) to no donor state outside the conduction band (Fig. 9h) upon gradual introduction of two atop $\mathrm{H}$ atoms on the edges of the $\mathrm{In}-[\mathrm{OH}]-\mathrm{In}$ bridge structure. Similarly, the $Y$ configuration, which lacks dangling bonds, has a higher conduction-band edge than the $(1 \times 2) \mathrm{In}-[\mathrm{OH}]-$ In bridge (compare Figs. 9e\&f).

The oxygen adatom in the In- $[\mathrm{OH}]-$ In bridge (Fig. 9a$\mathrm{c}, \mathrm{f}-\mathrm{g})$ contributes three primary signatures to the PDOS. The first, a peak at around -7.0 to $-7.5 \mathrm{eV}$, is associated with the $\mathrm{O}-\mathrm{H}$ bond but contains minor In character. Interestingly, this peak does not appear in the In$\mathrm{OH}$ atop descriptions (Fig. 9c\&i), although an analogous peak with $\mathrm{P}$ character is seen for $\mathrm{P}-\mathrm{OH}$ (Fig. 9d). A second, shallower peak, associated with the In-O bond, is found for most of the surfaces near $-6 \mathrm{eV}$. Finally, there is a signature at around $-3 \mathrm{eV}$ that is predominantly oxygen-derived (for the $\mathrm{In}-\mathrm{OH}$ descriptions, this peak is closer to $-1.5 \mathrm{eV})$.

\section{DISCUSSION}

The (100) surface of $\mathrm{GaP}$ and $\mathrm{InP}$ is characteristic among the common low-energy surfaces in that it features $M-M$ surface dimerization. Our results suggest that adsorption of oxygen or hydroxyl is likely to break the surface $M-M$ dimerization that is characteristic of the (001) surface. Of the possible oxygen local bond configurations, two should dominate oxygen chemisorption and subsequent native oxide nucleation based on their relative stability: $M-\mathrm{O}-\mathrm{P}$ and $M-\mathrm{O}-M$. Notably, these same two topologies have been suggested based on x-ray photoemission spectra of $\operatorname{InP}(001)^{23}$, as well as calculations on $\mathrm{InP}$ nanowires ${ }^{65}$. Both topologies can also branch to form an additional $M-\mathrm{O}$ bond with some cost in energy. The strength of the $\mathrm{Ga}-\mathrm{O}$ bond relative to $\mathrm{In}-\mathrm{O}$ means GaP should show a clear thermodynamic preference for $M-\mathrm{O}-M$ over $M-\mathrm{O}-\mathrm{P}$, whereas $\mathrm{InP}$ is approximately even for the two (with $M-\mathrm{O}-\mathrm{P}$ perhaps slightly favored over $M-\mathrm{O}-M)$. Although this does not necessarily predict which bulk oxide will grow natively under equilibrium conditions, it does suggest that the initial prenucleation of sub-monolayer oxide growth on $\mathrm{GaP}(001)$ should favor a $\mathrm{Ga}_{2} \mathrm{O}_{3}$-like structure, whereas a combination of $\mathrm{In}_{2} \mathrm{O}_{3}$ - and $\mathrm{InPO}_{4}$-like structures are expected on $\operatorname{InP}(001)$. On other surfaces where $M-M$ dimers are not present, $M-\mathrm{O}-\mathrm{P}$ bonds should dominate. These conclusions are consistent with suggestions from several experiments on $\mathrm{InP}$ and $\mathrm{GaP}$ oxidation ${ }^{23,24,26,66-69}$. However, we should note that for the (100) surface, $M-\mathrm{O}-$ $M$ is an exclusively surface-adsorbed topology, whereas $M-\mathrm{O}-\mathrm{P}$ features oxygen incorporated in the subsurface. It is therefore likely that the kinetics of oxygen incor- 


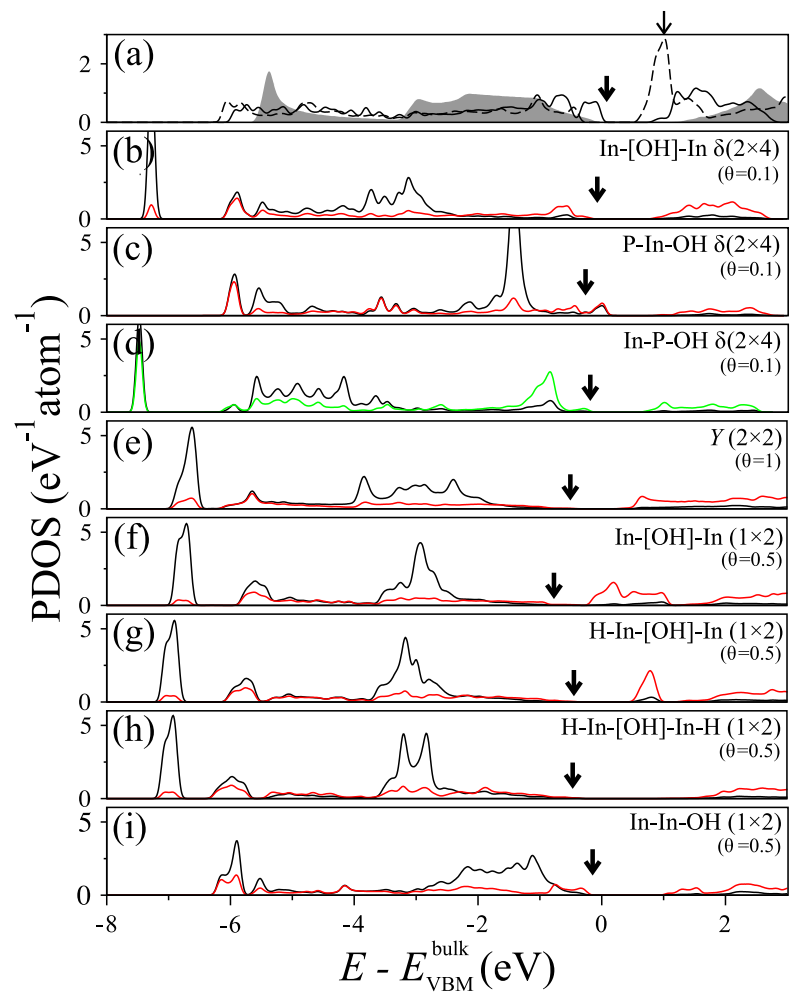

FIG. 9. (Color online) PDOS for surface atoms of local $\operatorname{InP}(001)$ bond topologies for several of the $\mathrm{OH}$-decorated surfaces. Black/red/green curves represent $\mathrm{O} / \mathrm{In} / \mathrm{P}$ densities of states. Highest occupied surface states are shown as arrows. See caption of Fig. 6 for an explanation of (a). The remaining configurations represent: (b-d) sites \#5, \#3, and \#1 on the mixed-dimer $\delta(2 \times 4)$ (see Fig. 1); (e) the $Y$ dense-coverage surfaces (see Fig. $2 \mathrm{~b})$; (f-h) the $(1 \times 2)$ dimer lattice with a surface bridge hydroxyl and with no atop $\mathrm{H}$, with one atop $\mathrm{H}$, and with two atop $\mathrm{H}$, respectively; and (i) the $(1 \times 2)$ dimer lattice with an atop $\mathrm{OH}$. Energies are referenced to the valence-band maximum of bulk InP.

poration may play a significant role in determining oxide morphology, yet our simulations do not provide further information on subsurface diffusion barriers for oxygen or gallium/indium. As such, we hesitate to make definitive conclusions about oxide growth beyond the first monolayer based on thermodynamics alone. It is worth emphasizing that kinetics are known to be relevant for oxide growth on III-V semiconductors at ambient temperatures, where conditions far from equilibrium lead to amorphization or formation of metastable compositions that differ from the high-temperature variants ${ }^{24,26,27,29}$. This means that in real samples, lowest-energy oxygen topologies are likely to coexist with the additional topologies we study here. A detailed investigation of the kinetics of oxygen incorporation presents an opportunity for future study that may offer additional insight.

Results for the hydroxide show the $M-[\mathrm{OH}]-M$ bridge and $M-\mathrm{OH}$ atop as the dominant bonding configurations for highly $M$-rich surfaces (we can neglect the atop $\mathrm{P}_{-}$
$\mathrm{OH}$ if we assume that the surface phosphorus concentration is small). In this case, there is little distinction between $\mathrm{GaP}$ and $\mathrm{InP}$ in terms of the relative stability of these two. For $M-\mathrm{OH}$, significant stabilization is seen for neighboring pairs of atop $\mathrm{OH}$ groups, which can hydrogen bond with one another. Further stabilization of the atop $\mathrm{OH}$ is expected in solution, where water can directly hydrogen-bond with the adsorbate. Comparison of the formation energies for the oxide and hydroxide (Figs. 3 and 4) points to the energetic stability of the hydroxide relative to the oxide. Barring other thermodynamic contributions, this indicates a driving force for hydroxylation not only of the clean surface in alkaline solution, but also of the oxide surface via protonation.

Signatures in the electronic structure point to important implications for the $M-\mathrm{O}-M$ topology (Figs. 7 and 8). In particular, $M-\mathrm{O}-M$ can give rise to hole traps if the bond is strained, either by symmetry constraints or by activation of surface modes, or if the $M$ atoms on either side of the oxygen bridge are bound to a monovalent adsorbate $(\mathrm{H}$ or $\mathrm{OH})$. In light of the connection between the $M-\mathrm{O}-M$ bond geometry and the character of the valence band maximum, activation of surface phonon modes or fluctuations in the surface bond chemistry could lead to significant reductions in the surface hole mobility of an oxygen-decorated $\mathrm{GaP} / \mathrm{InP}$ photoelectrode. In addition, we find that the $M-\mathrm{O}-M$ and $M-[\mathrm{OH}]-M$ bridge topologies, as well as $M-M$ surface dimers, can introduce shallow donor states in the gap if there are unsaturated bonds on the edge $M$ atoms. It is worth emphasizing that in those instances where the character and position of the band edges are significantly affected, as we see especially for the $M-\mathrm{O}-M$ bond topology, the oxygen adsorbate and its immediate environment will largely determine the surface chemistry. This points to the importance of including oxygen-derived surface adsorbates in realistic models of $\mathrm{GaP}$ or $\mathrm{InP}$ surfaces.

The existence of the hole trap on the $M-\mathrm{O}-M$ bond topology provides a compelling possible connection to the observed loss of cathodic protection in $\mathrm{GaP} / \mathrm{InP}$-based electrodes in operating photoelectrochemical cells. This conclusion derives in part from experiments which found that cathodic corrosion requires exciton generation, and that simple injection of electrons via application of current in the dark did not lead to electrode degradation ${ }^{8}$. This implies that the presence of holes is key to the corrosion mechanism, which probably derives from a shift in the local potential from regional hole buildup that is not compensated by the electrolyte solution. Furthermore, no hole traps are observed for $M-[\mathrm{OH}]-M$ bridges, so it is reasonable to conclude that the introduction of hole traps may be connected to proton donation from the low-energy hydroxylated surface, from which $M-\mathrm{O}-$ $M$ bridges might be formed. Notably, hole traps have been connected to photocorrosion in other semiconductor materials ${ }^{70-72}$.

The uniformity of our results across the tested samples allows us to map features in the electronic structure to 
bond topologies, as well as to specific bond geometries or bond chemistries within those topologies. This suggests that a local topological model may reproduce many of the key characteristics of the early stages of surface oxide and hydroxide formation. This is supported by the qualitative analysis of the common structural features in our tested surfaces (Fig. 5), which were found to closely correlate with analogous characteristics of the amorphous and crystalline bulk oxide phases. For example, the bond angle and length distributions are in near perfect agreement with the bulk amorphous oxide $\mathrm{In}_{2} \mathrm{O}_{3}$, despite the fact that our models consider only monolayer or submonolayer oxygen coverage. For the $M-\mathrm{O}-M$ topology, the observed dependence of the band-edge states on lattice strain and local bond chemistry suggests that the key functional characteristics of the surface should be reproduced as long as these features are accounted for in the model. We should mention that there are instances where only some of the local structural characteristics of the bulk phases are reproduced by our simulations, namely, $M-\mathrm{O}-\mathrm{P}$ and $M-[\mathrm{OH}]-M$. Fortunately, for these topologies, the electronic structure in the valence band region generally carries a weaker dependence on the local structure. Since the chemical properties of the surface are ultimately largely dependent on the electronic structure near the HOMO/LUMO levels (corresponding to the valence and conduction band edges), these should be preserved in the local model. We point out that in the case of InP, the connection between electronic structure and local morphology was successfully exploited in Ref. ${ }^{29}$ to categorize amorphous native surface oxides based on $\mathrm{x}$ ray photoelectron spectral features similar to those found for crystalline bulk phases. An analogous approach has also been proposed to assign oxides of $\mathrm{GaAs}^{73}$.

\section{SUMMARY AND CONCLUSIONS}

In summary, we have performed extensive total-energy calculations on a wide variety of known and theoretically obtained oxide- and hydroxide-decorated surfaces of $\mathrm{InP} / \mathrm{GaP}(001)$. Although realistic oxygen-rich polar III-V surfaces are expected to show a richly complex surface morphology, our results demonstrate that essential features in the morphology and electronic structure can be rationalized based on topological and geometric char- acteristics of the local adsorbate environment. This offers a simple and straightforward strategy for modeling the interplay between surface morphology, electronic structure, and chemistry in realistic oxygen- and hydroxyldecorated (001) surfaces of $\mathrm{GaP}$ and $\mathrm{InP}$. In addition, the local nature of the model means many of our conclusions are likely to be transferable to surfaces other than (001), as well as step edges and boundaries, provided similar bond topologies exist. We identify four principal adsorbate bond topologies $(M-\mathrm{O}-M, M-\mathrm{O}-\mathrm{P}, M-[\mathrm{OH}]-$ $M, M-\mathrm{OH})$, each of which carries distinctive signatures in the electronic structure. The $M-\mathrm{O}-M$ topology can significantly impair the mobility of hole carriers when strained mechanically, thermally, or by high oxygen coverage, or when the dangling bonds on the $M$ atoms are saturated. The resulting hole traps may contribute to the observed photocorrosion of $\mathrm{GaP} / \mathrm{InP}$-based electrode materials in operating photoelectrochemical cells. The electronic signatures associated with each of the bond topologies should be detectable using surface-sensitive spectroscopic methods (including X-ray techniques), providing a means for experimental identification of local structural topologies in native oxide films and offering insight into microscopic mechanisms of surface oxide nucleation. Such efforts are currently underway. By mapping the surface electronic structure to the adsorbate binding configuration, our results may augment experimental efforts towards improved III-V-based electrodes for photoelectrochemistry, as well as for additional applications where the formation of native oxide and hydroxide films may impact desired functionality.

\section{ACKNOWLEDGMENTS}

The authors acknowledge helpful discussions with J. Turner, T. Deutsch, H. Wang, and K. Kim (NREL), and with V. Lordi and W. Choi (LLNL). Funding was provided by the U.S. Department of Energy Fuel Cell Technologies Program. T. O. acknowledges additional funding from the Laboratory Directed Research and Development (LDRD) Grant 11-ERD-73. Computing support came from the LLNL Institutional Computing Grand Challenge program. This work was performed under the auspices of the U.S. Department of Energy by LLNL under Contract DE-AC52-07NA27344.
1 U.S. Department of Energy, DOE BESAC Report (2008).

2 A. Fujishima and K. Honda, Nature, 238, 37 (1972).

3 N. S. Lewis and D. G. Nocera, PNAS, 103, 15729 (2006).

4 J. Turner et al., Int. J. Energy Res., 32, 379 (2008).

${ }^{5}$ O. Khaselev and J. A. Turner, Science, 280, 425 (1998).

6 T. G. Deutsch, J. L. Head, and J. A. Turner, J. Electrochem. Soc., 155, B903 (2008).

7 H. Wang and J. A. Turner, ECS Trans., 2, 125 (2007).
8 T. G. Deutsch, C. A. Koval, and J. A. Turner, J. Phys. Chem. B, 110, 25297 (2006).

9 J. Vigneron, M. Herlem, E. M. Khoumri, and A. Etcheberry, Appl. Surf. Sci., 201, 51 (2002).

${ }^{10}$ H. Lewerenz and K. Schulte, Electrochim. Acta, 47, 2639 (2002).

11 O. Khaselev and J. A. Turner, J. Electrochem. Soc., 145, 3335 (1998). 
12 S. Menezes, B. Miller, and K. J. Bachmann, J. Vac. Sci. Technol. B, 1, 48 (1983).

13 A. Heller, Science, 223, 1141 (1984).

${ }^{14}$ H. J. Lewerenz, D. E. Aspnes, B. Miller, D. L. Malm, and A. Heller, J. Am. Chem. Soc., 104, 3325 (1982).

${ }^{15}$ W. E. Spicer, I. Lindau, P. Skeath, C. Y. Su, and P. Chye, Phys. Rev. Lett., 44, 420 (1980).

16 A. Heller, B. Miller, H. J. Lewerenz, and K. J. Bachmann, J. Am. Chem. Soc., 102, 6555 (1980).

17 B. C. Wood, T. Ogitsu, and E. Schwegler, J. Photon. Energy, 1, 016002 (2011).

18 M. Tomkiewicz and J. M. Woodall, Science, 196, 990 (1977).

19 M. J. Price and S. Maldonado, J. Phys. Chem. C, 113, 11988 (2009).

20 M. Szklarczyk and J. O. Bockris, J. Phys. Chem., 88, 5241 (1984).

21 O. Henrion, A. Klein, and W. Jaegermann, Surf. Sci., 457, L337 (2000).

22 N. Gayathri, S. Izvekov, and G. A. Voth, J. Chem. Phys., 117, 872 (2002).

${ }^{23}$ G. Chen, S. B. Visbeck, D. C. Law, and R. F. Hicks, J. Appl. Phys., 91, 9362 (2002).

24 M. Milojevic, C. L. Hinkle, E. M. Vogel, and R. M. Wallace, Fundamentals of III-V Semiconductor MOSFETs, edited by S. Oktyabrksy and P. D. Ye (Springer Science + Business Media LLC, 2010).

${ }^{25}$ G. Hollinger, G. Hughes, F. J. Himpsel, J. L. Jordan, and J. F. Morar, Surf. Sci., 168, 617 (1986).

26 C. W. Wilmsen, J. Vac. Sci. Technol., 19, 279 (1981).

27 J. Zemek, O. Baschenko, and M. Tyzykhov, Thin Solid Films, 224, 141 (1993).

${ }^{28}$ O. Pluchery, Y. J. Chabal, and R. L. Opila, J. Appl. Phys., 94, 2707 (2003).

29 G. Hollinger, E. Bergignat, J. Joseph, and Y. Robach, J. Vac. Sci. Technol. A, 3, 2082 (1985).

${ }^{30}$ C. W. Wilmsen, K. M. Geib, R. Gann, J. Costello, G. Hryckowian, and R. J. Zeto, J. Vac. Sci. Technol. B, 3, 1103 (1985).

31 Giannozzi, P. et al., J. Phys. Condens. Matt., 21, 395502 (2009).

32 J. P. Perdew, K. Burke, and M. Ernzerhof, Phys. Rev. Lett., 77, 3865 (1996).

33 D. Vanderbilt, Phys. Rev. B, 41, 7892 (1990).

34 S. V. Levchenko and A. M. Rappe, Phys. Rev. Lett., 100, 256101 (2008).

35 W. G. Schmidt, Appl. Phys. A, 75, 89 (2002).

36 O. Tereshchenko, D. Paget, P. Chiaradia, E. Placidi, J. Bonnet, F. Wiame, and A. Taleb-Ibrahimi, Surf. Sci., 600, 3160 (2006).

37 O. Pulci, K. Lüdge, P. Vogt, N. Esser, W. Schmidt, W. Richter, and F. Bechstedt, Comp. Mater. Sci., 22, 32 (2001).

38 W. Schmidt, J. Bernholc, and F. Bechstedt, Appl. Surf. Sci., 166, 179 (2000).

39 L. Li, Q. Fu, C. H. Li, B.-K. Han, and R. F. Hicks, Phys. Rev. B, 61, 10223 (2000).
40 L. Li, B.-K. Han, Q. Fu, and R. F. Hicks, Phys. Rev. Lett., 82, 1879 (1999).

${ }^{41}$ P. Vogt, T. Hannappel, S. Visbeck, K. Knorr, N. Esser, and W. Richter, Phys. Rev. B, 60, R5117 (1999).

42 A. M. Frisch, W. G. Schmidt, J. Bernholc, M. Pristovsek, N. Esser, and W. Richter, Phys. Rev. B, 60, 2488 (1999).

43 W. Schmidt and F. Bechstedt, Surf. Sci., 409, 474 (1998).

44 P. Vogt, K. Lüdge, M. Zorn, M. Pristovsek, W. Braun, W. Richter, and N. Esser, J. Vac. Sci. Technol. B, 18, 2210 (2000).

45 S. Froyen and A. Zunger, J. Vac. Sci. Technol. B, 9, 2176 (1991).

46 Y. Wu, M. K. Y. Chan, and G. Ceder, Phys. Rev. B, 83, 235301 (2011).

47 G. P. Srivastava, J. L. Martins, and A. Zunger, Phys. Rev. B, 31, 2561 (1985).

48 M. Dionízio Morieira, P. Venezuela, and T. M. Schmidt, Nanotech., 19, 065203 (2008).

49 H. Ibach, Physics of Surfaces and Interfaces (Springer Berlin Heidelberg, 2006).

50 S. Froyen and A. Zunger, Phys. Rev. Lett., 66, 2132 (1991).

51 R. C. L. Mooney, Acta Crystallogr., 9, 113 (1956).

${ }^{52}$ M. Marezio, Acta Crystallogr., 20, 723 (1966).

53 D. F. Mullica, G. W. Beall, and W. O. Milligan, J. Inorg. Nucl. Chem., 41, 277 (1979).

54 H. Nakae, K. Kihara, M. Okuno, and S. Hirano, Z. Kristallogr., 210, 746 (1995).

55 S. Geller, J. Chem. Phys., 33, 676 (1960).

56 J. D. Scott, Am. Mineral., 56, 355 (1971).

57 F. Utsuno et al., Thin Solid Films, 496, 95 (2006).

58 D. Pulver, C. W. Wilmsen, D. Niles, and R. Kee, J. Vac. Sci. Technol. B, 19, 207 (2001).

59 R. C. L. Mooney-Slater, Acta Crystallogr., 14, 1140 (1961).

60 X. Tang and A. Lachgar, Inorg. Chem., 37, 6181 (1998).

${ }^{61}$ L. Thierry, P. Capucine, and F. Gérard, C. R. Acad. Sci. II C, 1, 667 (1998).

62 J. Badro, J. P. Itíe, and A. Polian, Eur. Phys. J. B, 1, 265 (1998).

63 Supplementary information.

64 P. Chye, C. Su, I. Lindau, C. Garner, P. Pianetta, and W. Spicer, Surf. Sci., 88, 439 (1979).

${ }^{65}$ T. M. Schmidt, Appl. Phys. Lett., 89, 123117 (2006).

66 Y. Mizokawa, O. Komoda, and S. Miyase, Thin Solid Films, 156, 127 (1988).

67 J. F. Wager, K. M. Geib, C. W. Wilmsen, and L. L. Kazmerski, J. Vac. Sci. Technol. B, 1, 778 (1983).

68 H. Iwasaki, Y. Mizokawa, R. Nishitani, and S. Nakamura, Jap. J. Appl. Phys., 17, 1925 (1978).

69 R. Nishitani, H. Iwasaki, Y. Mizokawa, and S. Nakamura, Jap. J. Appl. Phys., 17, 321 (1978).

${ }^{70}$ K. W. Frese, M. J. Madou, and S. R. Morrison, J. Phys. Chem., 84, 3172 (1980).

71 H. Gerischer, J. Vac. Sci. Technol., 15, 1422 (1978).

72 P. V. Kamat, Chem. Rev., 93, 267 (1993).

${ }^{73}$ G. Lucovsky, J. Vac. Sci. Technol., 19, 456 (1981). 